\title{
QUASINORMAL FAMILIES AND PERIODIC POINTS
}

\author{
WALTER BERGWEILER
}

Dedicated to Larry Zalcman on his 60th Birthday

\begin{abstract}
Let $n \geq 2$ be an integer and $K>1$. By $f^{n}$ we denote the $n$-th iterate of a function $f$. Let $\mathcal{F}$ be the family of all functions $f$ holomorphic in some domain such that $\left|\left(f^{n}\right)^{\prime}(\xi)\right| \leq K$ whenever $f^{n}(\xi)=\xi$. We show that $\mathcal{F}$ is quasinormal of order 1 . If $K$ is sufficiently small, then $\mathcal{F}$ is normal. We also show that if $f$ is a transcendental entire function, then $f$ has a sequence $\left(\xi_{k}\right)$ of periodic points of period $n$ such that $\left(f^{n}\right)^{\prime}\left(\xi_{k}\right) \rightarrow \infty$ as $k \rightarrow \infty$.
\end{abstract}

\section{INTRODUCTION AND MAIN RESULTS}

Let $D \subset \mathbb{C}$ be a domain and let $f: D \rightarrow \mathbb{C}$ be a holomorphic function. The iterates $f^{n}: D_{n} \rightarrow \mathbb{C}$ of $f$ are defined by $D_{1}:=D, f^{1}:=f$ and $D_{n}:=f^{-1}\left(D_{n-1}\right)$, $f^{n}:=f^{n-1} \circ f$ for $n \in \mathbb{N}, n \geq 2$. Note that $D_{2}=f^{-1}\left(D_{1}\right) \subset D=D_{1}$ and thus $D_{n+1} \subset D_{n} \subset D$ for all $n \in \mathbb{N}$.

A point $\xi \in D$ is called a periodic point of period $n$ of $f$ if $\xi \in D_{n}$ and $f^{n}(\xi)=\xi$, but $f^{m}(\xi) \neq \xi$ for $1 \leq m \leq n-1$. A periodic point of period 1 is called a fixed point. The periodic points of period $n$ are thus the fixed points of $f^{n}$ which are not fixed points of $f^{m}$ for any $m$ less than $n$. Let $\xi$ be a periodic point of period $n$ of $f$. We say that $\xi$ is repelling if $\left|\left(f^{n}\right)^{\prime}(\xi)\right|>1$.

The periodic points play an important role in complex dynamics. For example, the Julia set of a rational or entire function, which is defined as the set where the iterates fail to be normal, is the closure of the set of repelling periodic points.

The following result is due to M. Essén and S. Wu [11, Theorem 1].

Theorem A. Let $D \subset \mathbb{C}$ be a domain and let $\mathcal{F}$ be the family of all holomorphic functions $f: D \rightarrow \mathbb{C}$ for which there exists $n=n(f)>1$ such that $f^{n}$ has no repelling fixed point. Then $\mathcal{F}$ is normal.

Without the word "repelling" the same authors had proved this result earlier in [10]. We mention that the results by Essén and $\mathrm{Wu}[10,11]$ answered a question of L. Yang [20, Problem 8].

We note that in Theorem A the condition that $f^{n}$ has no repelling fixed point cannot be replaced by the condition that $f$ has no periodic point of period $n$. In fact, the family $\mathcal{F}=\{n z\}_{n \in \mathbb{N}}$ is not normal at 0 , and the functions in $\mathcal{F}$ do not have periodic points of period greater than one. Also, $\mathcal{F}=\left\{-z+a z^{2}\right\}_{a \in \mathbb{C} \backslash\{0\}}$ is not normal at 0 , and the functions in $\mathcal{F}$ do not have periodic points of period 2. It was shown in [3, Theorem 2] that - in a suitable sense - non-normal sequences of holomorphic functions which fail to have periodic points of some period greater than one always arise from these examples.

Moreover, the following result was proved in [3, Theorem 3].

2000 Mathematics Subject Classification. Primary 30D45; Secondary 30D05, 37F10.

This research was supported by the German-Israeli Foundation for Scientific Research and Development (G.I.F.), grant no. G-643-117.6/1999. 
Theorem B. Let $D \subset \mathbb{C}$ be a domain and let $\mathcal{F}$ be the family of all holomorphic functions $f: D \rightarrow \mathbb{C}$ for which there exists $n=n(f)>1$ such that $f$ has no repelling periodic point of period $n$. Then $\mathcal{F}$ is quasinormal.

Recall here that a family $\mathcal{F}$ of functions holomorphic in a domain $D$ is called quasinormal (cf. $[8,14,19])$ if for each sequence $\left(f_{k}\right)$ in $\mathcal{F}$ there exists a subsequence $\left(f_{k_{j}}\right)$ and a finite set $E \subset D$ such that $\left(f_{k_{j}}\right)$ converges locally uniformly in $D \backslash E$. If the cardinality of the exceptional set $E$ can be bounded independently of the sequence $\left(f_{k}\right)$, and if $q$ is the smallest such bound, then we say that $\mathcal{F}$ is quasinormal of order $q$.

Our first result is an improvement of Theorem B.

Theorem 1.1. Let $K>1$ and let $D \subset \mathbb{C}$ be a domain. Let $\mathcal{F}$ be the family of all holomorphic functions $f: D \rightarrow \mathbb{C}$ for which there exists $n=n(f)>1$ such that $\left|\left(f^{n}\right)^{\prime}(\xi)\right| \leq K$ for every periodic point $\xi$ of period $n$ of $f$. Then $\mathcal{F}$ is quasinormal of order 1 .

The following results are closely connected to Theorems A and B.

Theorem C. Let $f$ be a transcendental entire function and let $n \in \mathbb{N}, n \geq 2$. Then $f^{n}$ has infinitely many fixed points.

Theorem D. Let $f$ be a transcendental entire function and let $n \in \mathbb{N}, n \geq 2$. Then $f$ has infinitely many repelling periodic points of period $n$.

Theorem C is due to P. C. Rosenbloom [18] while Theorem D can be found in [4, Theorem 1]. We mention that already P. Fatou [12, p. 345] had proved that the second iterate of a transcendental entire function has a fixed point; he had used this result to show [12, p. 348-350] that the Julia set of an entire transcendental function is always non-empty.

The connection between Theorems A and B on the one hand and Theorems $\mathrm{C}$ and $\mathrm{D}$ on the other hand is given by a heuristic principle attributed to A. Bloch which relates normal families and entire functions. This principle says that the family of all holomorphic functions with a certain property is likely to be normal if all entire functions with this property are constant. More generally, one may expect normality or at least quasinormality, if there are only "few" entire functions with this property. For an excellent discussion of Bloch's principle we refer to the book by J. Schiff [19] and two papers by L. Zalcman [21, 22].

The following sharpening of Theorem D can be considered as an analogue of Theorem 1.1 for entire functions according to Bloch's principle.

Theorem 1.2. Let $f$ be a transcendental entire function and let $n \in \mathbb{N}, n \geq 2$. Then $f$ has a sequence $\left(\xi_{k}\right)$ of periodic points of period $n$ such that $\left(f^{n}\right)^{\prime}\left(\xi_{k}\right) \rightarrow \infty$ as $k \rightarrow \infty$.

Theorem 1.1 shows in particular that if $K>1$, then the family $\mathcal{F}_{K}$ of all holomorphic functions $f$ for which there exists $n \geq 2$ such that $\left|\left(f^{n}\right)^{\prime}(\xi)\right| \leq K$ for all fixed points $\xi$ of $f^{n}$ is quasinormal. Considering the family $\mathcal{G}=\left\{a z^{2}\right\}_{a \in \mathbb{C} \backslash\{0\}}$ we see that $\mathcal{F}_{K}$ fails to be normal if $K \geq 4$. Indeed, for $f(z)=a z^{2} \in \mathcal{G}$ the fixed points of $f^{2}$ are given by $\xi=0$ and $\xi=\omega / a$ where $\omega$ is a third root of unity, and we have $\left(f^{2}\right)^{\prime}(0)=0$ and $\left(f^{2}\right)^{\prime}(\omega / a)=4$ so that $\mathcal{G} \subset \mathcal{F}_{4}$. Clearly $\mathcal{G}$ is not normal at 0 , and thus $\mathcal{F}_{K}$ is not normal if $K \geq 4$.

It does not seem unlikely that $\mathcal{F}_{K}$ is normal if $K<4$. In this direction we have the following result. 
Theorem 1.3. For each integer $n$ greater than 1 there exists a constant $K_{n}>1$ with the following property: if $D \subset \mathbb{C}$ is a domain and $\mathcal{F}$ is a family of holomorphic functions $f: D \rightarrow \mathbb{C}$ such that $\left|\left(f^{n}\right)^{\prime}(\xi)\right| \leq K_{n}$ for all fixed points $\xi$ of $f^{n}$, then $\mathcal{F}$ is normal.

The example mentioned above shows that the conclusion of Theorem 1.3 does not hold for $K_{n}=2^{n}$. Possibly it holds for each $K_{n}<2^{n}$.

Acknowledgments. I thank Jürgen Grahl for useful comments on an early version of this paper.

\section{Preliminary Lemmas}

We shall build on the ideas developed in $[3,10,11]$. As in these papers, one of the central tools comes from the Ahlfors theory of covering surfaces; see [1], [13, Chapter 5] or [15, Chapter XIII] for an account of this theory. The idea to use the Ahlfors theory to prove the existence of repelling periodic points is due to I. N. Baker [2] who had used it to show that such points are dense in the Julia set of an entire transcendental function. For a survey of further applications of the Ahlfors theory in complex dynamics we refer to [6].

Given a holomorphic function $f: D \rightarrow \mathbb{C}$ and a Jordan domain $V \subset \mathbb{C}$, we say that $f$ has a simple island over $V$ if $f^{-1}(V)$ has a component $U$ with $\bar{U} \subset D$ such that $\left.f\right|_{U}: U \rightarrow V$ is bijective. Such a component $U$ is then called a simple island over $V$. We will often use the trivial observation that if $f$ has a simple island $U$ over $V$ and if $V^{\prime}$ is a Jordan domain contained in $V$, then $f$ has a simple island $U^{\prime} \subset U$ over $V^{\prime}$.

The result from the Ahlfors theory that we need is the following Lemma 2.1. Besides the references already mentioned, we refer to [5] for a proof.

Lemma 2.1. Let $D \subset \mathbb{C}$ be a domain and let $D_{1}, D_{2}$ and $D_{3}$ be Jordan domains with pairwise disjoint closures. Let $\mathcal{F}$ be a family of functions holomorphic in $D$ which is not normal. Then there exists a function $f \in \mathcal{F}$ which has an island over $D_{1}, D_{2}$ or $D_{3}$.

We mention that this result has a counterpart for entire functions according to the principle by Bloch already mentioned: if $f$ is a non-constant entire function and if $D_{1}, D_{2}, D_{3}$ are Jordan domains with pairwise disjoint closures, then $f$ has a simple island over at least one of these domains. This result (and similarly Lemma 2.1) does not hold if we take only two domains $D_{1}$ and $D_{2}$, as shown by the example $f(z)=\cos z, D_{1}=D\left(-1, \frac{1}{2}\right), D_{2}=D\left(1, \frac{1}{2}\right)$, with $D(a, r):=\{z \in \mathbb{C}:|z-a|<r\}$. A rather simple, but for the purposes of this paper quite important observation is that if $f$ is a polynomial, then we need only two domains $D_{1}$ and $D_{2}$ in the above statement. More generally, this holds for polynomial-like mappings $f$.

By definition, if $U, V \subset \mathbb{C}$ are Jordan domains with $\bar{U} \subset V$, and if $f: U \rightarrow V$ is a proper holomorphic map (of degree $d$ ), then the triple $(f, U, V)$ is called a polynomial-like map (of degree $d$ ). The basic result about polynomial-like maps (see [7, Theorem VI.1.1] or [9, Theorem 1]) says that polynomial-like maps are quasiconformally conjugate to polynomials (of the same degree), but we shall not need this result. We mention that the concept of a polynomial-like mapping, created by A. Douady and J. H. Hubbard [9], was also used in [3, 4, 10, 11] to prove the existence of (repelling) fixed points and periodic points. 
Lemma 2.2. Let $(f, U, V)$ be a polynomial-like map of degree $d$ and let $D_{1}$ and $D_{2}$ be Jordan domains with pairwise disjoint closures contained in $V$. Then there exist two domains $U_{1}, U_{2} \subset U$ which are simple islands over $D_{1}$ or $D_{2}$.

Note that $U_{1}$ and $U_{2}$ need not be islands over the same domain. We allow the possibility that $U_{1}$ is an island over $D_{1}$ and $U_{2}$ is an island over $D_{2}$, or vice versa.

Proof of Lemma 2.2. We denote by $V_{1}, \ldots, V_{m}$ the components of $f^{-1}\left(D_{1}\right)$ and by $W_{1}, \ldots, W_{n}$ the components of $f^{-1}\left(D_{2}\right)$. Let $\mu_{j}$ be the degree of the proper map $\left.f\right|_{V_{j}}: V_{j} \rightarrow D_{1}$ and let $\nu_{j}$ be the degree of the proper map $\left.f\right|_{W_{j}}: W_{j} \rightarrow D_{2}$. Then $d=\sum_{j=1}^{m} \mu_{j}=\sum_{j=1}^{n} \nu_{j}$. By the Riemann-Hurwitz formula, $f$ has $d-1$ critical points in $U$, and of these critical points there are $\mu_{j}-1$ critical points in $V_{j}$ and $\nu_{j}-1$ critical points in $W_{j}$. Thus

$$
d-1 \geq \sum_{j=1}^{m}\left(\mu_{j}-1\right)+\sum_{j=1}^{n}\left(\nu_{j}-1\right)=\sum_{j=1}^{m} \mu_{j}+\sum_{j=1}^{n} \nu_{j}-m-n=2 d-m-n .
$$

This yields $m+n \geq d+1$. Since $f$ has $d-1$ critical points, at least two of the domains $V_{j}, W_{j}$ do not contain a critical point and thus are simple islands.

The following lemma is simple and well-known.

Lemma 2.3. Let $0<\delta<\frac{\varepsilon}{2}$ and let $U \subset D(a, \delta)$ be a simply-connected domain. Let $f: U \rightarrow D(a, \varepsilon)$ be holomorphic and bijective. Then $f$ has a fixed point $\xi$ in $U$ which satisfies $\left|f^{\prime}(\xi)\right| \geq \varepsilon / 4 \delta$.

Proof. We consider the inverse function $g: D(a, \varepsilon) \rightarrow U$ of $f$. It follows easily from Rouché's theorem that $g$ has a fixed point $\xi \in U \subset D(a, \delta)$. The function

$$
h(z)=\frac{1}{2 \delta}\left(g\left(\frac{\varepsilon}{2} z+\xi\right)-\xi\right)
$$

then maps the unit disk $D(0,1)$ into itself and satisfies $h(0)=0$. From Schwarz's lemma we can now deduce that $1 \geq\left|h^{\prime}(0)\right|=\varepsilon\left|g^{\prime}(\xi)\right| / 4 \delta$. The conclusion follows since $f^{\prime}(\xi)=1 / g^{\prime}(\xi)$.

We recall some elementary graph theoretic notions used in [3] (and implicit in $[10,11])$. For a set $V$ and a set $E \subset V \times V$ we call the pair $G=(V, E)$ a digraph. The elements of $V$ are called vertices and those of $E$ are called edges. In contrast to the usual terminology we allow loops; that is, we do not exclude edges $e$ of the form $e=(v, v)$ with $v \in V$.

We call $w=\left(v_{0}, v_{1}, \ldots, v_{n}\right) \in V^{n+1}$ a closed walk of length $n$ if $v_{0}=v_{n}$ and $\left(v_{k-1}, v_{k}\right) \in E$ for $k=1, \ldots, n$. Note that we do not assume that $v_{j} \neq v_{k}$ for $0 \leq j<k \leq n-1$. We call a closed walk $w=\left(v_{0}, v_{1}, \ldots, v_{n}\right)$ primitive if there does not exists $p \in \mathbb{N}, 1 \leq p<n$, such that $p \mid n$ and $v_{j}=v_{k}$ for all $j, k$ satisfying $p \mid(j-k)$. A primitive closed walk is thus a closed walk which is not obtained by running through a closed walk of smaller length several times. Finally recall that the outdegree of a vertex $v$ is defined to be the cardinality of $\{u \in V:(v, u) \in E\}$.

As in $[3, \S 5]$ we shall use the following lemma.

Lemma 2.4. Let $q, n \in \mathbb{N}, q \geq 6, n \geq 2$. Let $G$ be a digraph with $q$ vertices such that the outdegree of each vertex is a least $q-2$. Then $G$ contains a primitive closed walk of length $n$. 
We shall also use the following well-known result (see [8, p. 131] or [19, Proposition A.2]) about quasinormal families, whose simple proof we include for completeness.

Lemma 2.5. Let $\mathcal{F}$ be a family of functions holomorphic in a domain D. Suppose that $\mathcal{F}$ is quasinormal of order $q$. Let $\left(f_{k}\right)$ be a sequence in $\mathcal{F}$ and let $a_{1}, \ldots, a_{q} \in D$. If no subsequence of $\left(f_{k}\right)$ is normal at any of the points $a_{j}$, then $f_{k} \rightarrow \infty$ in $D \backslash\left\{a_{1}, \ldots, a_{q}\right\}$.

Proof. Since $\mathcal{F}$ is quasinormal of order $q$ and no subsequence of $\left(f_{k}\right)$ is normal at the points $a_{j}$, the sequence $\left(f_{k}\right)$ is normal in $D \backslash\left\{a_{1}, \ldots, a_{q}\right\}$. If a subsequence of $\left(f_{k}\right)$ would tend to a finite limit function in $D \backslash\left\{a_{1}, \ldots, a_{q}\right\}$, then, by the maximum principle, this subsequence would be locally bounded and thus normal in $D$. Thus $f_{k} \rightarrow \infty$ in $D \backslash\left\{a_{1}, \ldots, a_{q}\right\}$.

The following result [19, Theorem A.6] is a simple consequence of Lemma 2.5.

Lemma 2.6. Let $\mathcal{F}$ be a family of functions holomorphic in a domain D. Suppose that $\mathcal{F}$ is quasinormal of order $q$. If the functions in $\mathcal{F}$ are bounded at $q+1$ points of $D$, then $\mathcal{F}$ is normal.

\section{Proof of Theorem 1.1}

First we proof that $\mathcal{F}$ is quasinormal of order at most 5 . The argument in this part of the proof is essentially the same as in [3]. We refer to this paper for further details.

Assuming that $\mathcal{F}$ is not quasinormal of order at most 5 we find six points $a_{1}, \ldots, a_{6} \in D$ and a sequence $\left(f_{k}\right)$ in $\mathcal{F}$ such that no subsequence of $\left(f_{k}\right)$ is normal in a neighborhood of any $a_{j}$. We choose $\varepsilon<\min _{i \neq j}\left|a_{i}-a_{j}\right|$ and $\delta<\varepsilon / 4 K$. For fixed $k$ we consider the digraph $G=(V, E)$ whose vertices are the $a_{j}$ and whose edges are all pairs $\left(a_{i}, a_{j}\right)$ for which $f_{k}$ has a simple island over $D\left(a_{j}, \varepsilon\right)$ which is contained in $D\left(a_{i}, \delta\right)$. It follows from Lemma 2.1 that if $k$ is large enough, then the outdegree of each vertex is at least 4 . Lemma 2.4 now shows that if $n \geq 2$, then $G$ contains a primitive closed walk $\left(a_{i_{0}}, a_{i_{1}}, \ldots, a_{i_{n}}\right)$ of length $n$. Thus $D\left(a_{i_{n-1}}, \delta\right)$ contains a simple island $U_{n-1}$ over $D\left(a_{i_{n}}, \varepsilon\right)$. Next $D\left(a_{i_{n-2}}, \delta\right)$ contains a simple island over $D\left(a_{i_{n-1}}, \varepsilon\right)$ and thus in particular a simple island $U_{n-2}$ over $U_{n-1}$. Inductively we find simple islands $U_{j} \subset D\left(a_{i_{j}}, \delta\right)$ over $U_{j+1}$, for $j=0,1, \ldots, n-2$. We deduce that $f^{n}$ maps $U_{0}$ bijectively onto $D\left(a_{i_{n}}, \varepsilon\right)$. Since $U_{0} \subset D\left(a_{i_{0}}, \delta\right)=D\left(a_{i_{n}}, \delta\right)$ we deduce from Lemma 2.3 that $f_{k}^{n}$ has a fixed point $\xi \in U_{0} \subset D\left(a_{i_{0}}, \delta\right)$ with $\left|\left(f_{k}^{n}\right)^{\prime}(\xi)\right| \geq \varepsilon / 4 \delta>K$. Since the walk $\left(a_{i_{0}}, a_{i_{1}}, \ldots, a_{i_{n}}\right)$ is primitive, this fixed point $\xi$ of $f_{k}^{n}$ is in fact a periodic point of period $n$ of $f_{k}$. This contradicts the assumption that $f_{k} \in \mathcal{F}$.

Hence $\mathcal{F}$ is quasinormal of order $q$ for some $q \leq 5$. We have to show that $q=1$ and thus assume that $q \geq 2$. As before there exist $q$ points $a_{1}, \ldots, a_{q} \in D$ and a sequence $\left(f_{k}\right)$ in $\mathcal{F}$ such that no subsequence of $\left(f_{k}\right)$ is normal in a neighborhood of any $a_{j}$. From Lemma 2.5 we deduce that $f_{k} \rightarrow \infty$ in $D \backslash\left\{a_{1}, \ldots, a_{q}\right\}$.

Again we choose $\varepsilon<\min _{i \neq j}\left|a_{i}-a_{j}\right|$ and $\delta<\varepsilon / 4 K$. We also choose $R>$ $\max _{j}\left|a_{j}\right|+\varepsilon$ and find that if $k$ is sufficiently large, then $\left|f_{k}(z)\right|>R$ for $\left|z-a_{j}\right|=\delta$ and $j=1, \ldots, q$. On the other hand, since no subsequence of $\left(f_{k}\right)$ is normal at $a_{j}$, we have $\left|f_{k}(z)\right|<R$ for some $z \in D\left(a_{j}, \delta\right)$ is $k$ is large. Thus $f_{k}^{-1}(D(0, R))$ has a component $U_{j} \subset D\left(a_{j}, \delta\right)$ for $j=1, \ldots, q$, provided that $k$ is large enough. Clearly $\left(\left.f_{k}\right|_{U_{j}}, U_{j}, D(0, R)\right)$ is a polynomial-like map. 
By Lemma 2.2 there exists two simple islands over $D\left(a_{1}, \varepsilon\right)$ or $D\left(a_{2}, \varepsilon\right)$ contained in $U_{1}$, and two further simple islands over $D\left(a_{1}, \varepsilon\right)$ or $D\left(a_{2}, \varepsilon\right)$ contained in $U_{2}$. Without loss of generality we may assume that two of these four simple islands are over $D\left(a_{1}, \varepsilon\right)$; that is, we have two simple islands $V_{1}, W_{1}$ over $D\left(a_{1}, \varepsilon\right)$ which are contained in $U_{1} \cup U_{2}$. Using Lemma 2.2 we now find for $j=2,3, \ldots, n-1$ domains $V_{j}, W_{j} \subset U_{2}$ which are simple islands over $V_{j-1}$ or $W_{j-1}$, and finally domains $V_{n}, W_{n} \subset U_{1}$ which are simple islands over $V_{n-1}$ or $W_{n-1}$. Then $f^{n}$ maps $V_{n}$ bijectively onto $D\left(a_{1}, \varepsilon\right)$. Lemma 2.3 shows that $f^{n}$ has a fixed point $\xi \in$ $V_{n}$ with $\left|\left(f^{n}\right)^{\prime}(\xi)\right|>K$. Moreover, $f^{j}(\xi) \in V_{n-j} \cup W_{n-j} \subset U_{2} \subset D\left(a_{2}, \delta\right)$ for $j=1,2, \ldots, n-2$, and this implies that $\xi$ is in fact a periodic point of period $n$ provided that $n \geq 3$.

We now consider the case that $n=2$. If $V_{1}, W_{1}$ are both contained in $U_{2}$, then the above argument also works for $n=2$; that is, the fixed point $\xi \in V_{2} \subset U_{1}$ of $f^{2}$ constructed above satisfies $f(\xi) \in V_{1} \cup W_{1} \subset U_{2}$ and thus is not a fixed point of $f$, but a periodic point of period 2. Next we consider the case that $V_{1}, W_{1}$ are both contained in $U_{1}$. Since $V_{1}$ is a simple island over $D\left(a_{1}, \varepsilon\right)$ and $W_{1} \subset U_{1} \subset D\left(a_{1}, \varepsilon\right)$, we see that $V_{1}$ contains a simple island $X$ over $W_{1}$. Since $f^{2}$ maps $X$ bijectively onto $D\left(a_{1}, \varepsilon\right)$, Lemma 2.2 again yields a fixed point $\xi \in X$ of $f^{2}$ with $\left|\left(f^{2}\right)^{\prime}(\xi)\right|>K$. Since $f(\xi) \in W_{1}$ and $X \cap W_{1} \subset V_{1} \cap W_{1}=\emptyset$, we have $f(\xi) \neq \xi$ so that $\xi$ has period 2. Finally we consider the case that both $U_{1}$ and $U_{2}$ contain only one island over $D\left(a_{1}, \varepsilon\right)$, say $V_{1} \subset U_{1}$ and $W_{1} \subset U_{2}$. Then $U_{1} \cup U_{2}$ also contains two simple islands $X_{1}, Y_{1}$ over $D\left(a_{2}, \varepsilon\right)$. We may assume that one of them is contained in $U_{1}$ and one is contained in $U_{2}$, say $X_{1} \subset U_{1}$ and $Y_{1} \subset U_{2}$, since otherwise we are in one of the situations already considered. Now $X_{1}$ contains a simple island $Z$ over $W_{1}$ and thus $f^{2}$ maps $Z$ bijectively onto $D\left(a_{1}, \varepsilon\right)$. As before we deduce from Lemma 2.2 that $f$ has a periodic point $\xi \in Z$ of period 2 with $\left|\left(f^{2}\right)^{\prime}(\xi)\right|>K$.

We have thus obtained a contradiction to the assumption that $q \geq 2$ in all cases.

\section{Proof of Theorem 1.2}

The basic idea is essentially the same as in $[3, \S 4]$. Let $\left(c_{k}\right)$ be a sequence tending to $\infty$ such that $\left(f\left(c_{k}\right)\right)$ is bounded. Define $f_{k}: \mathbb{C} \rightarrow \mathbb{C}$ by $f_{k}(z)=f\left(c_{k} z\right) / c_{k}$. Then $f_{k}(0) \rightarrow 0$ and $f_{k}(1) \rightarrow 0$.

Suppose now that the conclusion does not hold. Then there exists $K>1$ such that $\left|\left(f^{n}\right)^{\prime}(\xi)\right| \leq K$ for all periodic points $\xi$ of period $n$ of $f$. We note that if $\xi$ is a periodic point of $f_{k}$ of period $n$, then $c_{k} \xi$ is a periodic point of $f$ of the same period, and $\left(f_{k}^{n}\right)^{\prime}(\xi)=\left(f^{n}\right)^{\prime}\left(c_{k} \xi\right)$. Thus the sequence $\left(f_{k}\right)$ is quasinormal of order 1 by Theorem 1.1. Since $\left(f_{k}\right)$ is bounded at 0 and 1 , Lemma 2.6 yields that $\left(f_{k}\right)$ is normal.

On the other hand, it is not difficult to see that $\left(f_{k}\right)$ is not normal at 0 . For example, this follows since $M(r, f):=\max _{|z|=r}|f(z)| \geq r^{2}$ for sufficiently large $r$ so that

$$
M\left(\frac{1}{\sqrt{\left|c_{k}\right|}}, f_{k}\right)=\frac{M\left(\sqrt{\left|c_{k}\right|}, f\right)}{\left|c_{k}\right|} \geq 1
$$

for large $k$, while $f_{k}(0) \rightarrow 0$. Thus no subsequence of $\left(f_{k}\right)$ can converge in a neighborhood of 0 . 


\section{Proof of Theorem 1.3}

The proof of Theorem 1.3 is based on the following lemma due to X. Pang and L. Zalcman [17, Lemma 2].

Lemma 5.1. Let $\mathcal{F}$ be a family of functions meromorphic on the unit disc, all of whose zeros have multiplicity at least $k$, and suppose that there exists $A \geq 1$ such that $\left|g^{(k)}(\xi)\right| \leq A$ whenever $g(\xi)=0, g \in \mathcal{F}$. Then if $\mathcal{F}$ is not normal there exist, for each $0 \leq \alpha \leq k$, a number $r \in(0,1)$, points $z_{j} \in D(0, r)$, functions $g_{j} \in \mathcal{F}$ and positive numbers $\rho_{j}$ tending to zero such that

$$
\frac{g_{j}\left(z_{j}+\rho_{j} z\right)}{\rho_{j}^{\alpha}} \rightarrow G(z)
$$

locally uniformly, where $G$ is a nonconstant meromorphic function on $\mathbb{C}$ such that the spherical derivative $G^{\#}$ of $G$ satisfies $G^{\#}(z) \leq G^{\#}(0)=k A+1$ for all $z \in \mathbb{C}$.

We shall only need the case $k=1$ of Lemma 5.1 . This special case can also be found in [16, Lemma 2].

We also require the following result of Essén and $\mathrm{Wu}[11$, Theorem 4].

Lemma 5.2. Let $f$ be a polynomial of degree at least 2 and let $n \geq 2$. Then $f^{n}$ has a repelling fixed point.

Proof of Theorem 1.3. We denote by $\mathcal{F}(D, n, K)$ the family of all functions $f$ holomorphic in $D$ such that $\left|\left(f^{n}\right)^{\prime}(\xi)\right| \leq K$ whenever $f^{n}(\xi)=\xi$. Note that this implies that $\left|f^{\prime}(\xi)\right| \leq \sqrt[n]{K}$ whenever $f(\xi)=\xi$. We fix $n \geq 2$ and suppose that there does not exist $K>1$ such $\mathcal{F}(D, n, K)$ is normal, in order to seek a contradiction. We may assume that $D$ is the unit disk.

For $m \in \mathbb{N}$ we choose a non-normal sequence $\left(f_{j}\right)$ in $\mathcal{F}(D, n, 1+1 / m)$. With $g_{j}(z):=f_{j}(z)-z$ we find that if $g_{j}(\xi)=0$, then $f_{j}(\xi)=\xi$ and thus

$$
\left|g_{j}^{\prime}(\xi)\right| \leq\left|f_{j}^{\prime}(\xi)\right|+1 \leq \sqrt[n]{1+\frac{1}{m}}+1 \leq 3 .
$$

Clearly, the sequence $\left(g_{j}\right)$ is also not normal. Applying Lemma 5.1 for $\alpha=k=1$ and $A=3$ we may assume, passing to a subsequence if necessary, that there exist $z_{j} \in D$ and $\rho_{j}>0$ such that $g_{j}\left(z_{j}+\rho_{j} z\right) / \rho_{j} \rightarrow G_{m}(z)$ for some entire function $G_{m}$ satisfying $G_{m}^{\#}(z) \leq G_{m}^{\#}(0)=4$ for all $z \in \mathbb{C}$. With $L_{j}(z)=z_{j}+\rho_{j} z$ we find that

$$
h_{j}(z):=L_{j}^{-1}\left(f_{j}\left(L_{j}(z)\right)\right)=\frac{f_{j}\left(z_{j}+\rho_{j} z\right)-z_{j}}{\rho_{j}}=\frac{g_{j}\left(z_{j}+\rho_{j} z\right)}{\rho_{j}}+z \rightarrow G_{m}(z)+z .
$$

With $F_{m}(z):=G_{m}(z)+z$ we thus have $h_{j}(z) \rightarrow F_{m}(z)$ as $j \rightarrow \infty$. It follows that $h_{j}^{n}(z) \rightarrow F_{m}^{n}(z)$. The assumption that $f_{j} \in \mathcal{F}(D, n, 1+1 / m)$ implies that $h_{j} \in \mathcal{F}\left(L_{j}^{-1}(D), n, 1+1 / m\right)$; that is, $\left|\left(h_{j}^{n}\right)^{\prime}(\xi)\right| \leq 1+1 / m$ whenever $h_{j}^{n}(\xi)=\xi$. We deduce that $F_{m} \in \mathcal{F}(\mathbb{C}, n, 1+1 / m)$. Since $G_{m}^{\#}(z) \leq G_{m}^{\#}(0)=4$ for all $z \in \mathbb{C}$, the sequence $\left(G_{m}\right)$ is normal. Hence $\left(F_{m}\right)$ is normal and we may assume without loss of generality that $F_{m} \rightarrow F$ for some non-constant entire function $F$. Since $F_{m} \in \mathcal{F}(\mathbb{C}, n, 1+1 / m)$ we conclude that $F \in \mathcal{F}(\mathbb{C}, n, 1)$; that is, $F$ is an entire function such that $F^{n}$ has no repelling fixed point. We deduce from Lemma 5.2 and Theorem $\mathrm{D}$ (or Theorem 1.2) that $F$ is a polynomial of degree 1 at most. Next we note that $\left|F_{m}^{\prime}(0)\right| \geq\left|G_{m}^{\prime}(0)\right|-1 \geq G_{m}^{\#}(0)-1=3$ and thus $\left|F^{\prime}(0)\right| \geq 3$. Hence $F$ has the form $F(z)=a z+b$ where $|a| \geq 3$. With $\xi:=b /(1-a)$ we obtain $F(\xi)=\xi$ and $\left|F^{\prime}(\xi)\right|=|a| \geq 3$, contradicting $F \in \mathcal{F}(\mathbb{C}, n, 1)$. 


\section{REFERENCES}

[1] L. V. Ahlfors, Zur Theorie der Überlagerungsflächen, Acta Math. 65 (1935), 157-194, and Collected Papers, Birkhäuser, Boston, Basel, Stuttgart, 1982, Vol. I, pp. 214-251.

[2] I. N. Baker, Repulsive fixpoints of entire functions, Math. Z. 104 (1968), 252-256.

[3] D. Bargmann and W. Bergweiler, Periodic points and normal families, Proc. Amer. Math. Soc. 129 (2001), 2881-2888.

[4] W. Bergweiler, Periodic points of entire functions: proof of a conjecture of Baker, Complex Variables Theory Appl. 17 (1991), 57-72.

[5] _ A new proof of the Ahlfors five islands theorem, J. Analyse Math. 76 (1998), 337347.

[6] The role of the Ahlfors five islands theorem in complex dynamics, Conform. Geom. Dyn. 4 (2000), 22-34.

[7] L. Carleson and T. W. Gamelin, Complex dynamics, Springer, New York, Berlin, Heidelberg, 1993.

[8] C.-T. Chuang, Normal families of meromorphic functions, World Scientific, Singapore, 1993.

[9] A. Douady and J. H. Hubbard, On the dynamics of polynomial-like mappings, Ann. Sci. École Norm. Sup. (4) 18 (1985), 287-343.

[10] M. Essén and S. Wu, Fix-points and a normal family of analytic functions, Complex Variables Theory Appl. 37 (1998), 171-178.

[11] _ Repulsive fixpoints of analytic functions with applications to complex dynamics, J. London Math. Soc. (2) 62 (2000), 139-149.

[12] P. Fatou, Sur l'itération des fonctions transcendantes entières, Acta Math. 47 (1926), 337360.

[13] W. K. Hayman, Meromorphic functions, Clarendon Press, Oxford, 1964.

[14] P. Montel, Leçons sur les familles normales des fonctions analytiques et leurs applications, Gauthier-Villars, Paris, 1927.

[15] R. Nevanlinna, Eindeutige analytische Funktionen, Springer, Berlin, Göttingen, Heidelberg, 1953.

[16] X. Pang, Shared values and normal families, Analysis 22 (2002), 175-182.

[17] X. Pang and L. Zalcman, Normal families and shared values, Bull. London Math. Soc. 32 (2000), 325-331.

[18] P. C. Rosenbloom, L'itération des fonctions entières, C. R. Acad. Sci. Paris 227 (1948), 382-383.

[19] J. L. Schiff, Normal families, Springer, New York, Berlin, Heidelberg, 1993.

[20] L. Yang, Some recent results and problems in the theory of value-distribution, in Proceedings of the Symposium on Value Distribution Theory in Several Complex Variables, (W. Stoll, ed.), Univ. of Notre Dame Press, Notre Dame Math. Lect. 12 (1992), 157-171.

[21] L. Zalcman, A heuristic principle in complex function theory, Amer. Math. Monthly 82 (1975), 813-817.

[22] _ Normal families: new perspectives, Bull. Amer. Math. Soc. (N.S.) 35 (1998), 215230 .

Mathematisches Seminar der Christian-Albrechts-Universität Zu Kiel, LudewigMeyn-Strasse 4, D-24098 Kiel, Germany

E-mail address: bergweiler@math.uni-kiel.de 